\title{
The development of a wireless LCP-based intracranial pressure sensor for traumatic brain injury patients
}

\author{
Preedipat Sattayasoonthorn ${ }^{1}$, Jackrit Suthakorn ${ }^{2}$, Sorayouth Chamnanvej, M.D ${ }^{3}$ \\ ${ }^{1}$ Department of Biomedical Engineering, Mahidol University, Thailand \\ ${ }^{2}$ Center for Biomedical and Robotics Technology, Mahidol University, Thailand \\ ${ }^{3}$ Department of Surgery, Mahidol University, Thailand
}

\begin{tabular}{l} 
Article Info \\
\hline Article history: \\
Received Jun 10, 2019 \\
Revised Oct 9, 2019 \\
Accepted Oct 21, 2019 \\
\hline Keywords: \\
Finite Element Method \\
Intracranial Pressure sensor \\
LCP \\
MEMS Fabrication \\
Simulation
\end{tabular}

Article Info

Article history:

Received Jun 10, 2019

Revised Oct 9, 2019

Keywords:

Finite Element Method LCP

Simulation

\begin{abstract}
Raised intracranial pressure (ICP) in traumatic brain injury (TBI) patients can lead to death. ICP measurement is required to monitor the condition of a patient and to inform TBI treatment. This work presents a new wireless liquid crystal polymer (LCP) based ICP sensor. The sensor is designed with the purpose of measuring ICP and wirelessly transmitting the signal to an external monitoring unit. The sensor is minimally invasive and biocompatible due to the mechanical design and the use of LCP. A prototype sensor and associated wireless module are fabricated and tested to demonstrate the functionality and performance of the wireless LCP-based ICP sensor. Experimental results show that the wireless LCP-based ICP sensor can operate in the pressure range of $0-60.12 \mathrm{mmHg}$. Based on repeated measurements, the sensitivity of the sensor is found to be 25.62 $\mu \mathrm{VmmHg}-1$, with a standard deviation of $\pm 1.16 \mu \mathrm{VmmHg}-1$. This work represents a significant step towards achieving a wireless, implantable, minimally invasive ICP monitoring strategy for TBI patients.
\end{abstract}

Copyright $@ 2020$ Institute of Advanced Engineering and Science. All rights reserved.

\section{Corresponding Author:}

Jackrit Suthakorn,

Center for Biomedical and Robotics Technology,

Faculty of Engineering,

Mahidol University,

25/25 Puttamonthon 4 Road, Salaya, Nakorn Pathom 73170, Thailand.

Email: jackrit.sut@mahidol.ac.th

\section{INTRODUCTION}

Traumatic brain injury (TBI) patients require specialist care, especially concerning intracranial pressure (ICP) monitoring. Increased ICP leads to severe brain damage, the two major consequences are brain shift and brain ischemia [1-3]. An excessive ICP can cause disability or death to a patient. A normal ICP range is between 5 to $10 \mathrm{mmHg}$ in adults, 3 to $7 \mathrm{mmHg}$ in children and 1.5 to $6 \mathrm{mmHg}$ in infants [4]. There are several techniques to measure and monitor ICP. Most conventional ICP measurement methods are invasive catheter-based procedures [5]. A number of clinical complications are encountered with catheterbased methods, such as infection and hemorrhage [6]. Additionally, patients with a catheter-based device are restricted to bed and lack mobility. A wireless minimally invasive ICP monitoring approach therefore is highly desirable to overcome the aforementioned limitations of catheter-based methods [7].

Most wireless ICP devices are designed to be an implantable system that is powered and can transfer data in order to minimize interference with the surrounding biological tissue and prevent the risk of infection. The design constraints are the size of device, the electrical supply of the device, and the device functionality [8]. There are two approaches to achieving a wireless implantable ICP sensor: the use of a passive sensor,or the use of an active sensor [8-10]. A passive sensor is a battery-free device, mostly they are based on inductive coupling which is suitable for small data packages. The principle of operation of 
a passive ICP sensor is as follows: an off-body coil is placed at an inductively coupling distance from the implanted coil which is inside the patient's head. A coupled magnetic field generates power to the sensor and transfers data from the sensor.

The data is transferred from the sensor by changing the impedance of the implanted coil that is detected by the off-body coil. The passive sensor has a low operating frequency and a short workable distance due to the requirement for inductive coupling [11]. Recently, several groups of researchers have focused on this approach. For example, the recent United States Food and Drug Administration (FDA) approved ICP sensor, AURA ${ }^{\mathrm{TM}}$ ICP Monitoring System (Branchpoint Technologies, Irvine, CA, USA) is a battery-free wireless ICP sensor in the market [12]. The AURA ${ }^{\mathrm{TM}}$ sensor receives power inductively from the AURA ${ }^{\mathrm{TM}}$ Monitor (Model T0011B) through the AURA ${ }^{\mathrm{TM}}$ Antenna (Model T0010A). The sensor has a catheter tip with a strain-gauge pressure sensor that extends into the brain parenchyma to measure ICP. The measured data is transferred to the antenna which connects to a monitor. Data connection is via low energy Bluetooth. The dimensions of the sensor are $7 \mathrm{~mm}$ thickness x $21 \mathrm{~mm}$ diameter of body and $25 \mathrm{~mm}$ long, with a catheter diameter of $2 \mathrm{~mm}$. This system has the advantage that the data receiver is provided to operate with other standard patient monitors. The monitoring duration is 29 days until the accuracy of the device shifts.

In [13] a wirelessly powered passive implant using a piezoresistive pressure sensor (Amphenol P162 NovaSensor, Amphenol Advanced Sensor, Fremont, CA, USA) was developed to measure ICP. The system consists of two parts; the implant and external reader. Inductive coupling was applied to the design for power transmission. This study shows that sufficient power was generated to activate the sensor for monitoring the pressure in the range of 0 to $28 \mathrm{mmHg}$. In a later study [14] the researchers developed a data transmission unit using a far-field antenna. The system was evaluated in a simulated biological environment. The pressure was measured over the range of -3 to $33 \mathrm{mmHg}$ and transmitted to the external receiver at a distance up to $1 \mathrm{~m}$.

In [15] the authors used a capacitive microelectromechanical system (MEMS) pressure sensor (Murata SCB10H-B012FB, Murata Electronics Oy, Vantaa, Finland) connecting to a $50 \mu \mathrm{m}$ spiral coil to form an LC tank. The coil rests on the skull and connects to the MEMS sensor in the subdural through RF coaxial cable. An increasing pressure induces a change in resonant frequency in LC tank, the changes can be detected by an external reader antenna. The operating pressure is in the range of 0 to $70 \mathrm{mmHg}$. In [16] a passive ICP sensor is made from two planar coils facing each other to create an LC tank. The sensor is designed to be placed under the dura mater. When pressure is applied on the top membrane, the two coils move closer resulting in a change of resonant frequency. A wearable readout system was proposed to detect the pressure changes over a wide frequency range of $35 \mathrm{MHz}-2.7 \mathrm{GHz}$. The author suggested that this reader is suitable for variable designs of sensors. Passive sensors have three main limitations for ICP measurement, such as the bulky size of the coil, magnetic resonance imaging (MRI) incompatibility, and short operating distances.

Active ICP sensors are battery-powered devices that contain the pressure sensor, data processing and wireless communication modules. This type of sensor allows the transmission of large data packages in shorter time periods over a longer distance and with better signal to noise ratio (SNR) than passive devices. There are two types of battery commonly use in implantable sensor; disposable and rechargeable. Rechargeable batteries are charged inductively from an external magnetic source. In [17] an analog wireless ICP sensor was developed using a capacitive MEMS pressure sensor (Murata Electronics Oy, Vantaa, Finland). The operating frequency is at the Industrial Scientific and Medical (ISM) band of $2.4 \mathrm{GHz}$. The change of pressure is detected by the sensor which modulates a $2.4 \mathrm{GHz}$ RF oscillator. The output signal is coupled to a planar inverted - $\mathrm{F}$ antenna. The transmitted data is detected by the receiver held in close distance to the head. A 3-V $220 \mathrm{mAh}$ lithium coin battery (CR2032, Panasonic, Secaucus, NJ, USA) supplies the power to all electrical components. The sensor's performance was evaluated in both in-vitro and in-vivo experiments.

In [18] the analog ICP sensor from [17] was implemented to be a digital device using a modified wireless development platform (eZ430-RF2500, Texas Instruments Incorporated, Dallas, TX, USA). The platform consists of a wireless radio (CC2500) and a microcontroller (MSP430) which connects to the capacitive MEMS sensor. When an increasing pressure is detected, the microcontroller measures the change in capacitance and sends the output through the wireless radio. The annular slot antenna is connected to the wireless radio to transmit the signal to the external readout unit. The size of the digital sensor is $22 \mathrm{~mm}$ width x $26 \mathrm{~mm}$ length $\mathrm{x} 10 \mathrm{~mm}$ thickness. The performance of the sensor was evaluated by measuring the dynamic ICP changes in a swine model.

In [19] the authors proposed a wireless sensor that measures ICP indirectly via air pressure. The system consists of an implantable sensing device and a portable data recorder. In the implantable sensing device, an air pouch sits in contact with the cerebrospinal fluid (CSF) and converts liquid pressure into air 
pressure. The changes in air pressure are detected by an air pressure sensor that connects to the data processing and transmitting part. This system was fabricated into a $3.04 \mathrm{~mm}$ x $2 \mathrm{~mm}$ system on chip (SoC). This device uses $3 \mathrm{~V} 50 \mathrm{mAh}$ battery and was tested in a hydrostatic environment. The operating pressure is in the range of -20 to $150 \mathrm{mmHg}$. The novelty of our work is to develop a biocompatible, implantable ICP sensor that is easy to fabricate with a low cost of manufacture that can operate during an MRI scan, none of the reviewed work above fully satisfy these requirements.

In this work, a wireless LCP-based ICP sensor is proposed to measure the change of ICP for TBI patients. The sensor is designed to measure the ICP in the skull and transmit the data to an external monitoring unit. The design of the sensor consists of three main parts: the pressure sensing unit, the data transmission unit and the power unit. The pressure sensing unit is designed to be placed on the dura mater to detect the changes of ICP, while the data transmission and power units will be integrated into a platform and placed externally on the skull. The sensor is designed to be a complete LCP package. Due to LCP's biocompatibility, it allows for a fully implantable device. The LCP-based pressure sensing unit is described in detail in our previous work $[20,21]$. A piezoresistive concept was adopted to design the sensing unit to operate in the 0 to $50 \mathrm{mmHg}$ pressure range. The LCP-based pressure sensing unit was fabricated using standard MEMS techniques and evaluated in a hydrostatic environment. The results showed an acceptable performance. The main contribution of the present work is the design and fabrication of an LCP-based ICP sensor that can measure and transmit the changes of ICP to the external unit wirelessly.

Regarding to this study, the aforementioned LCP pressure sensing unit is connected to a newly developed data transmission and power units to demonstrate the functionality of the wireless LCP-based ICP sensor. The sensor performance is evaluated under a pressure range of 0 to $50 \mathrm{mmHg}$. Design details of the wireless LCP sensor are given in Section 3. The experimental setup for evaluating the wireless LCP sensor is given in Section 4. Experimental results and analysis are given in Section 5.

\section{METHODOLOGY}

\subsection{Wireless LCP-based ICP sensor design overview}

The wireless LCP-based ICP sensor is designed to measure the change of ICP within the skull. The implantable sensor can detect the change of ICP and remotely transmit the data to the external monitoring unit through the scalp. The sensor structure consists of three main parts: the pressure sensing unit, the data transmission unit and the power unit as shown in Figure 1. The pressure sensing unit is designed to be placed under the skull to detect changes in ICP in the surrounding CSF. The data transmission and power units will be integrated into a platform and placed on the skull, underneath the scalp.

When ICP increases, the pressure sensing unit measures the changes in pressure and sends the sensor output to the data transmission unit. The processed data is transmitted to the external monitoring device. The power unit supplies power to the pressure sensing unit and other electrical circuits on the data transmission and power platform as shown in Figure 1. The size of the pressure sensing unit is smaller than the platform. The overall size of wireless LCP-based ICP sensor can be minimized in future design iterations.

The fabrication of the wireless LCP-based ICP sensor is designed to be biocompatible and implantable through the use of LCP. The pressure sensing unit was designed based on the piezoresistive concept and fabricated on an LCP membrane. The fabrication process and the characterization of LCP pressure sensing unit are described in [20]. LCP has high mechanical strength, low dielectric constant and various chemical resistance $[22,23]$. It is suitable for packaging the data transmission and power unit. The data transmission unit is designed based on $2.4 \mathrm{GHz}$ ISM bands. The data transmission unit uses Bluetooth communication to transmit data to the external monitoring unit, this design offers ease of interface and MRI compatibility [24]. The power unit presently requires a battery to supply power to the LCP pressure sensing unit and other circuits. An illustration of a conceptual design of the system is shown in Figure 2.

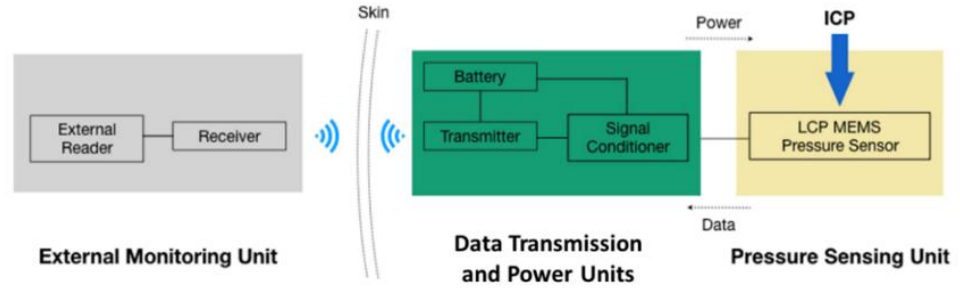

Figure 1. Overall operating principle of the wireless LCP-based ICP sensor 


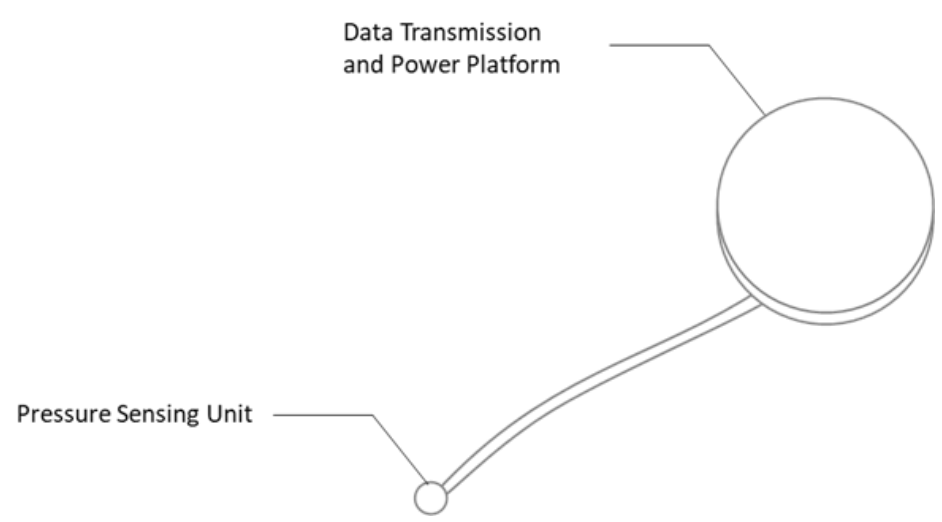

Figure 2. Illustration of a conceptual design of the proposed wireless LCP-based ICP sensor

\subsection{Wireless LCP-Based ICP Sensor Prototype}

The LCP pressure sensing unit contains four gold strain gauges with resistances between $449-535 \Omega$. The structure of this unit consists of an $8 \mathrm{~mm} \times 8 \mathrm{~mm} \times 100 \mu \mathrm{m}$ outer membrane and a $2 \mathrm{~mm} \times 2 \mathrm{~mm} \times 50 \mu \mathrm{m}$ sensing membrane above a bottom cavity. The outer membrane is bonded with a $10 \times 10 \mathrm{~mm} \times 100 \mu \mathrm{m}$ LCP membrane to seal the bottom cavity using adhesive. The LCP pressure sensing unit is connected to the data transmission and power platform through ribbon cables.

The platform of data transmission and power units consists of a Bluetooth to serial port module (HC-05), microcontroller (ATmega328/P, Atmel Corporation, USA), 16-bit analog-to-digital converter (ADC) (ADS 1115, Texas Instruments Incorporated, USA), Buck-Boost Converter (TPS63001, Texas Instruments Incorporated, USA) and 3.6 V lithium ion coin battery (LIR2450, $120 \mathrm{mAh}, 24.5 \mathrm{~mm}$ diameter). These components are presently assembled on FR4 board, and the total dimension of the platform is $30 \times 30 \mathrm{~mm}$.

Figure 3 shows the block diagram of the wireless LCP-based ICP sensor. The $3.6 \mathrm{~V} \mathrm{Li}$-on battery is used to power the LCP pressure sensing unit, ADC, microcontroller and Bluetooth module. The output voltage of the LCP pressure sensing unit is sent to the ADS 1115 and ATmega328/P controller respectively. The ADC resolution is 16 bits with full-scale range of $\pm 1.024 \mathrm{~V}$, which gives a resolution of $31.25 \mu \mathrm{V}$. The processed data is sent via serial communication to the HC-05 Bluetooth module. The HC-05 transmits data at 960 bps and $0 \mathrm{dBm} \mathrm{RF}$ transmit power (programmable up to $+4 \mathrm{dBm}$ ). The transmitted data is monitored with the serial monitor feature on Arduino 1.8.8 (Arduino, Somerville, USA). The prototype of the wireless LCP-based ICP sensor is shown in Figure 4.

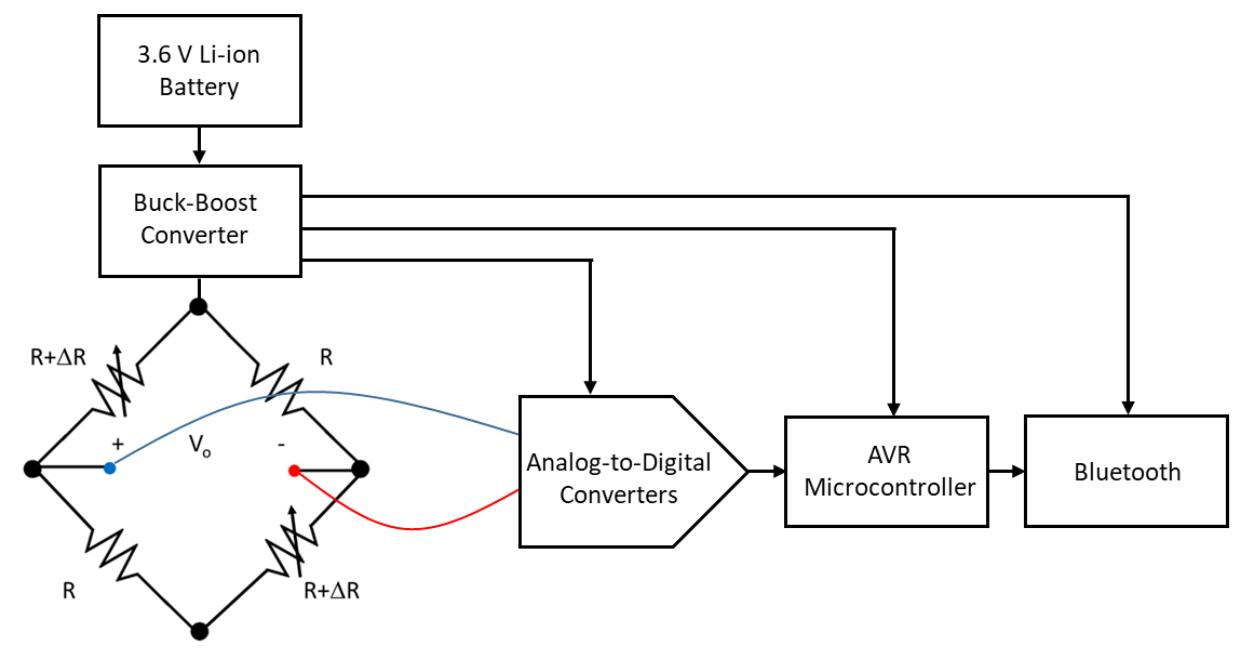

Figure 3. Block diagram of the wireless ICP measurement system 


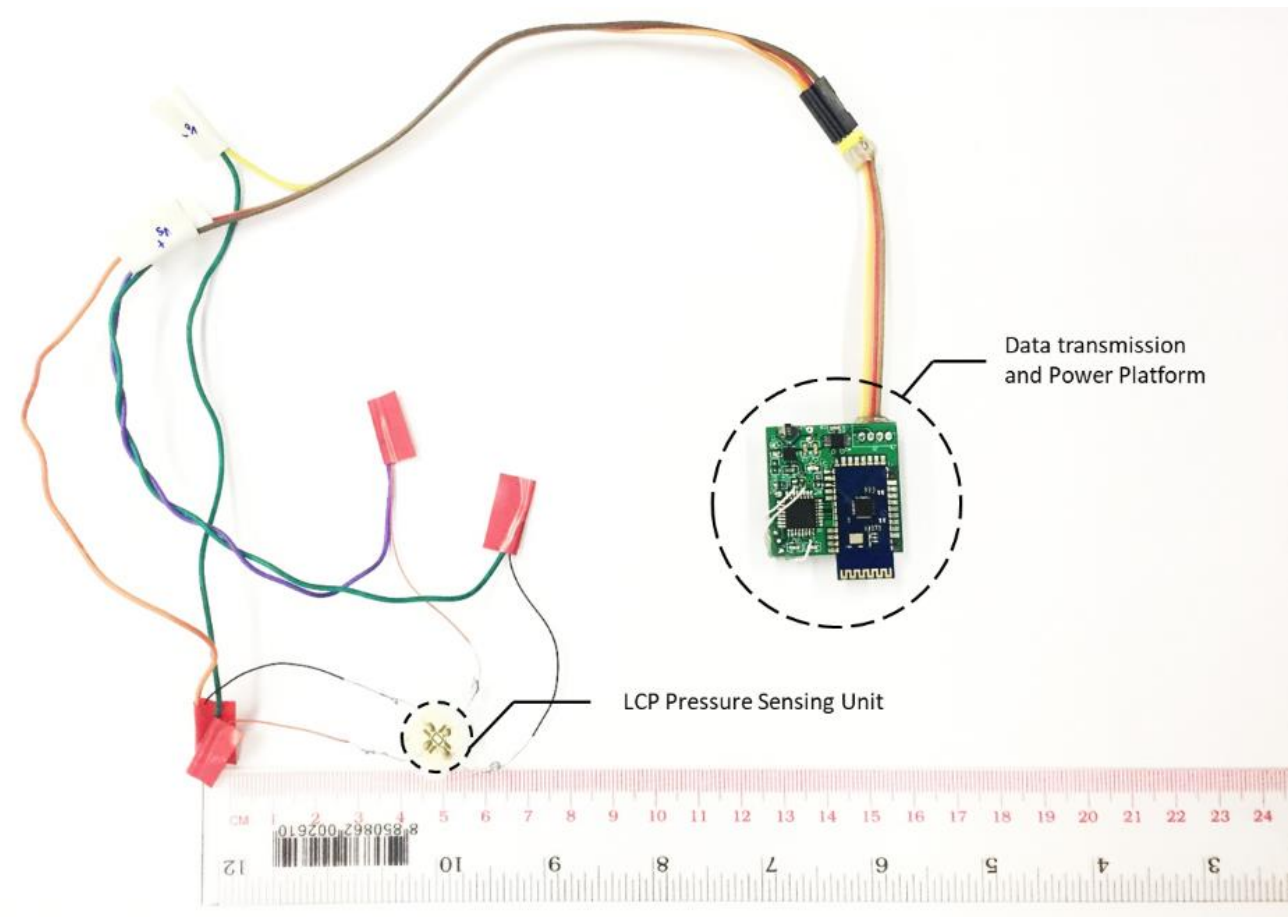

Figure 4. Prototype of the wireless LCP-based ICP sensor

\section{EXPERIMENT}

The wireless LCP-based ICP sensor is designed to operate in the pressure range of 0 to $50 \mathrm{mmHg}$. This experiment aims to mimic the pressures ranging from 0 to $50 \mathrm{mmHg}$ in ambient conditions by using an MTS Insight Electromechnical Testing systems (MTS Systems Corporation, Minnesota, USA). Theoretically, the pressure $(\mathrm{P})$ is generated by a force $(\mathrm{F})$ acting over a surface area $(\mathrm{A})$ that is in direct contact with the applied load. Applying this theory, the range of the force is calculated to correspond to the range of the pressure. In this case, $\mathrm{P}$ is the pressures ranging from 0 to $50 \mathrm{mmHg}$, and $\mathrm{A}$ is the $8 \times 8 \mathrm{~mm}^{2} \mathrm{LCP}$ sensing unit's area. The range of the corresponding force is therefore 0 to $0.5 \mathrm{~N}$. The MTS Insight system is set up for a compression test with a $100 \mathrm{~N}$ load cell and flat compression platens. TestWorks® software (MTS Systems Corporation, Minnesota, USA) is used to set up the input parameters and run the MTS Insight system on a personal computer. The parameters are the maximum force of $0.5 \mathrm{~N}$, the test speed of $5 \mu \mathrm{m} / \mathrm{s}$ and the sampling rate of $1 \mathrm{~Hz}$. A laptop is used to monitor the sensor output voltage that is measured and remotely transmitted on serial monitor. The clocks of these two computers are synchronized to correlate the time stamps. The overall measurement system is shown in Figure 5.

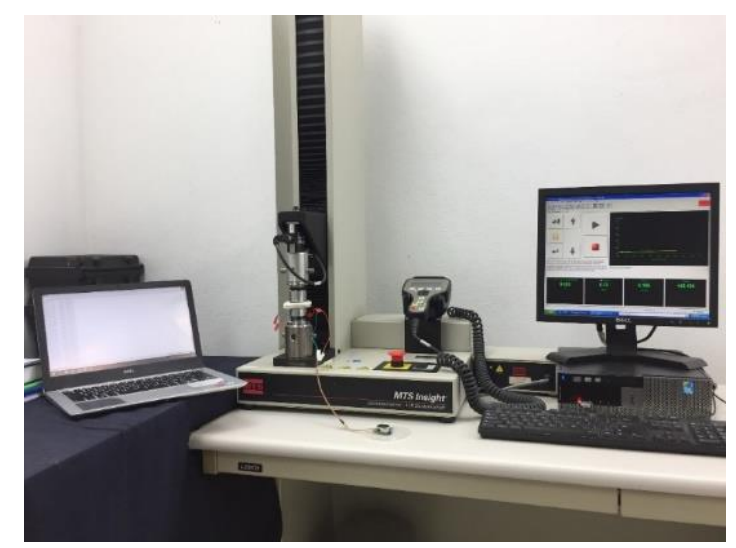

Figure 5. The experiment set up for testing wireless implantable ICP sensor 
The LCP pressure sensing unit is sandwiched between $5 \mathrm{~mm}$ thick silicone membranes. The silicone membranes act as cushions and distribute the pressure over the LCP pressure sensing unit. This sandwiched LCP pressure sensing unit is fixed on the compression platens as shown in Figure 6 (a) and (b). When the measurement starts, the serial monitor is run before starting the compression test on the MTS Insight system. The sensor output voltage is monitored and recorded on the serial monitor. When the MTS Insight system runs, the load applies the force ranging from 0 to $0.5 \mathrm{~N}$ at the testing speed of $5 \mu \mathrm{m} / \mathrm{s}$. The forces are acquired in TestWorks ${ }^{\circledR}$ software at the sampling rate of $1 \mathrm{~Hz}$. The applied load increases until the maximum force reaches the limitation at $0.5 \mathrm{~N}$. The applied forces and the time stamps at the starting and ending points are recorded via the MTS Insight system. The time stamp at the starting point of the MTS Insight system is aligned to the time stamp of serial monitor to correlate the forces and the sensor output voltages. The sensor output voltages were recorded for ten repeated measurements with the same experimental conditions.

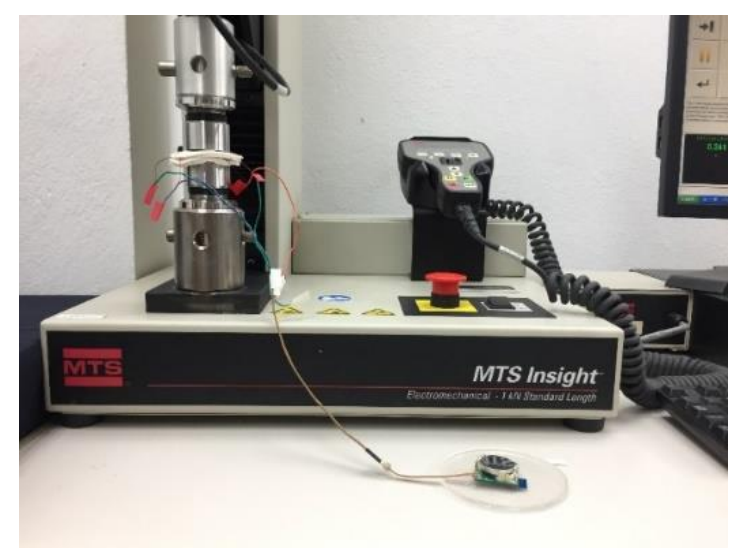

(a)

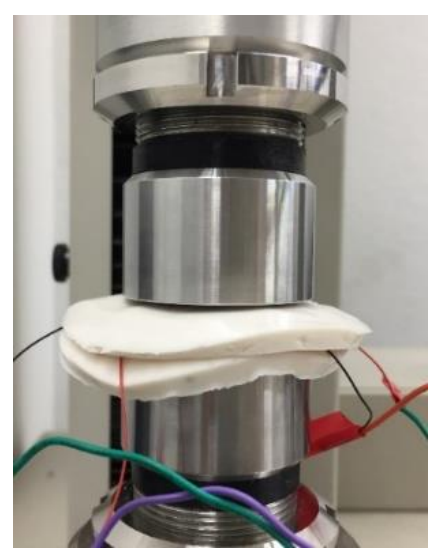

(b)

Figure 6. The wireless LCP-based ICP sensor under the compression test (a) the sandwiched LCP sensing unit connecting to the data transmission and power platform (b) the LCP pressure sensing unit in between two silicone membranes on compression platens.

\section{RESULTS}

The experiment was carried out to demonstrate the functionality and evaluate the performance of the wireless LCP-based ICP sensor. The sensor was subjected to loading and unloading conditions continuously. The recorded applied forces and the calculated pressures are plotted corresponding to time for ten repeated measurements as shown in Figure 7. From the graph, the pressure was applied from the base pressure (less than $1 \mathrm{mmHg}$ ) to the maximum pressure of $60.12 \mathrm{mmHg}$ within approximately 412 seconds. The sensor output voltages of each measurement are recorded and plotted with respect to the pressure as shown in Figure 8. The output voltage linearly increases with increasing pressure.

The sensitivity of the sensor is estimated from the slope of the best-fit line fitted to the data points for each repeated measurement, giving ten sensitivities. The average of these ten sensitivities over the pressure range of 0 to $60.12 \mathrm{mmHg}$ is found to be $25.62 \mu \mathrm{VmmHg}^{-1} \pm 1.16 \mu \mathrm{V}$ (average \pm standard deviation) as shown in Figure 9. The graph shows that the output voltage at $0 \mathrm{mmHg}$ is $0.951 \pm 94.34 \mu \mathrm{V}$. The repeatability of the sensor is obtained from the standard deviation of the repeated measurements at the pressures of $0,10,20,30,40$ and $50 \mathrm{mmHg}$ and presented as error bars as shown in Figure 9. The graph shows small error bars which indicates that the wireless implantable ICP sensor demonstrates good repeatability.

Figure 10 shows the residual of the best fit line of the sensor output voltage for all ten-measurement result. The residual captures the nonlinearity of the sensor alongside the measurement noise. There is no obvious structure to the residual plot which suggests the sensor output is very linear with respect to pressure, this is also supported by the $\mathrm{R}^{2}$ value of 0.984 , where an $\mathrm{R}^{2}$ value of 1 would indicate a perfectly linear relationship. The standard deviation of the measurement noise evaluated from Figure 10 is found to be 47.75 $\mu \mathrm{V}$ which indicates the meaningful resolution of the sensor is approximately $2 \mathrm{mmHg}$. The resolution of the sensor is limited by noise in this experiment but the noise may originate from the MTS Insight system which generates a fluctuating output signal at low forces. 


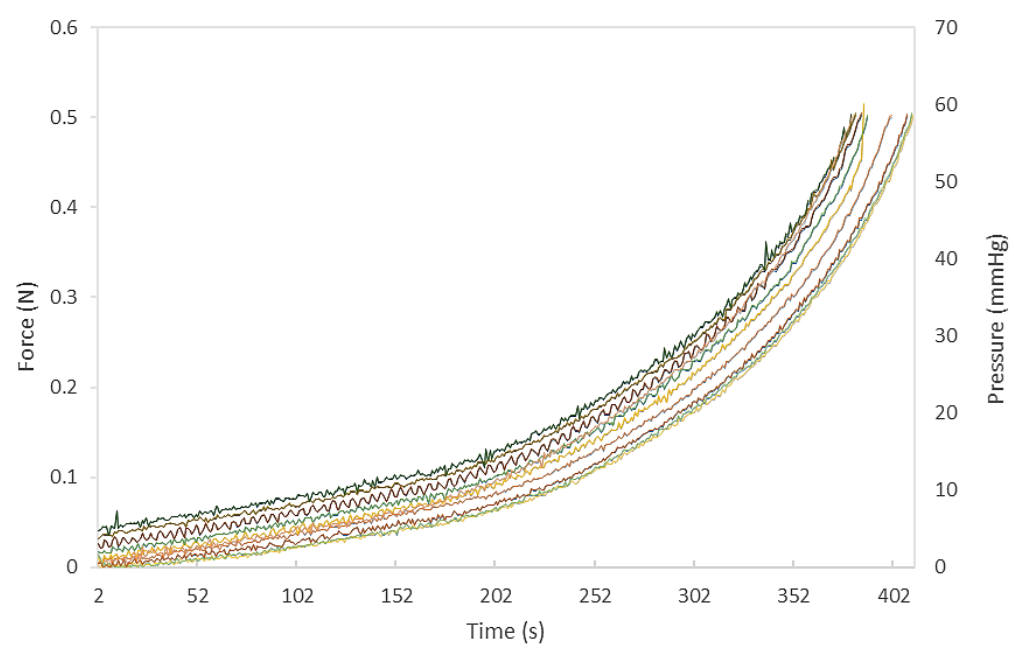

Figure 7. Graph of recorded applied force and pressure for ten repeated measurements

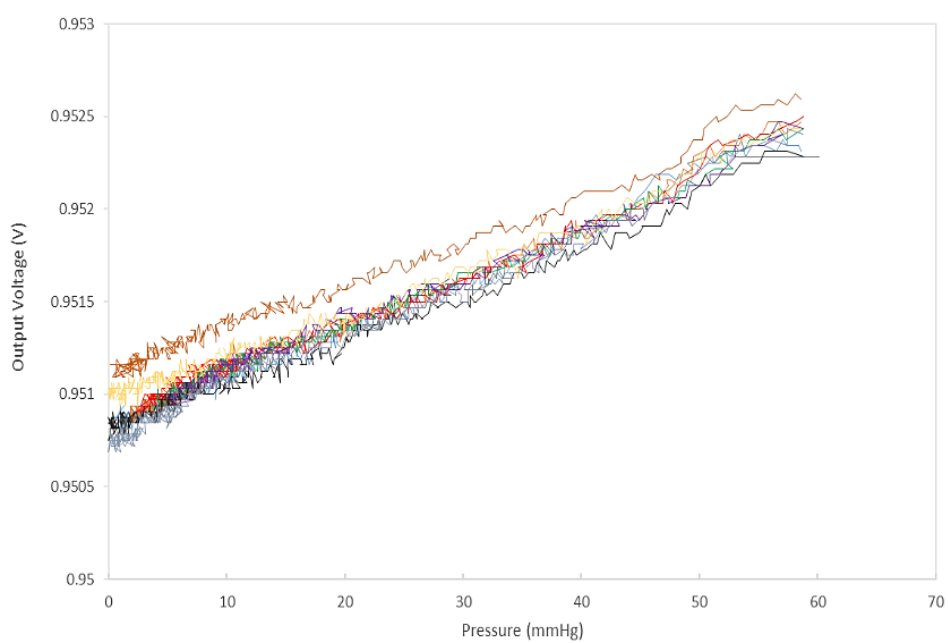

Figure 8. Graph of recorded sensor output voltage over the pressure range of 0 to $60.12 \mathrm{mmHg}$ for ten repeated measurements

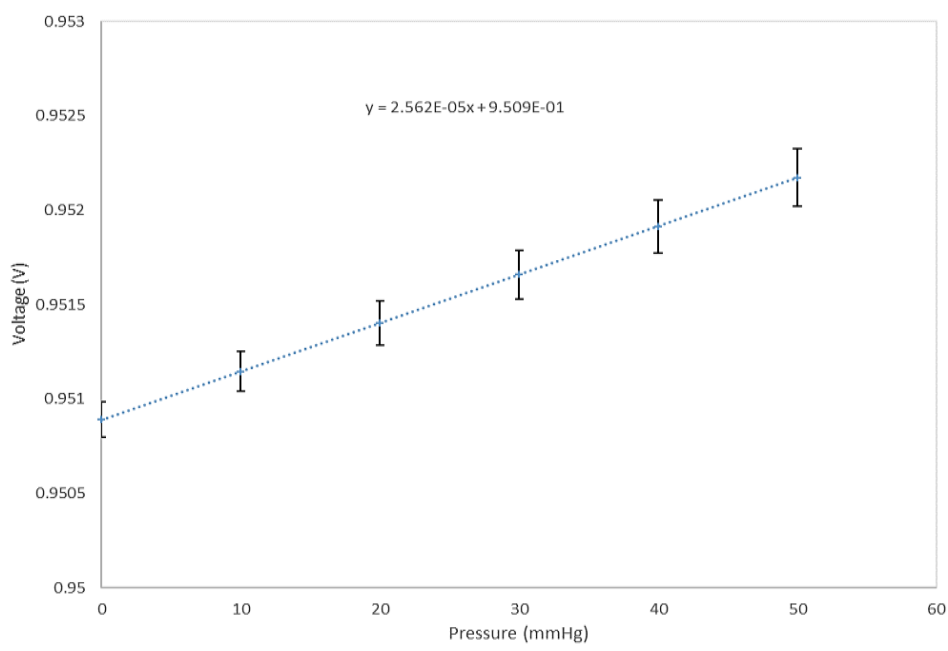

Figure 9. Graph of averaged output voltages under 0, 10, 20, 30, 40 and $50 \mathrm{mmHg}$ pressure range 


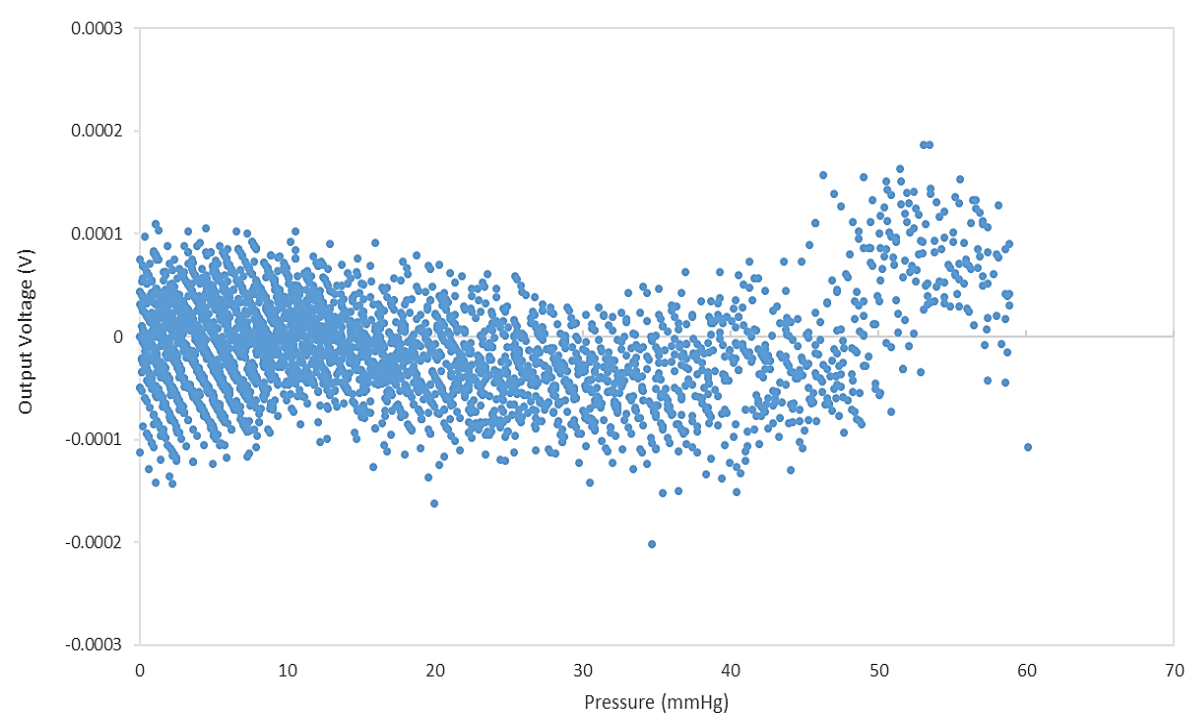

Figure 10. Plot of the residual of the best fit line to the measurement result of the sensor output voltage for all ten repeated measurements.

\section{DISCUSSION}

The goal of this work was to demonstrate the functionality and evaluate the performance of the developed wireless LCP-based ICP sensor. The experiment shows that the MEMS fabricated LCP pressure sensing unit can operate successfully in conjunction with the data transmission and power platform. The wireless LCP-based ICP sensor measured changes in pressure and transmitted the real-time data to the external monitoring device wirelessly. The sensor is able to measure the pressure from 0 to $60.12 \mathrm{mmHg}$ which is higher than the maximum design pressure. As shown in Figure 8, the output voltage increases linearly pressure. The sensitivity of the wireless LCP-based ICP sensor obtained from the slope in Figure 9 is lower than the simulated sensitivity reported in our previous work [20].

In future work the LCP pressure sensing unit will be refabricated to enhance the sensitivity. The data transmission and power platform will be further minimized by removing the battery holder, upgrading Bluetooth module and ADCs and encapsulated in LCP for packaging. The power consumption of the system can be improved in a number of ways, for example, enhancing the resistivity of the strain gauges in the LCP pressure sensing unit, implementing the data transmission unit with a low energy Bluetooth module and developing a power controller to configure the work mode of the sensor. The size of the data transmission and power unit could be further reduced in future work by replacing the data transmission and power platform with wireless energy harvesting technologies [25, 26].

\section{CONCLUSION}

A wireless LCP-based ICP sensor was proposed in this work. This study proves the concept and demonstrates the functionality of a wireless LCP-based pressure sensor to monitor the changes of ICP for TBI patients. The proposed prototype consists of a MEMS fabricated LCP pressure sensing unit and a data transmission and power platform. The sensor achieved a measurement sensitivity of $25.62 \mu \mathrm{VmmHg}-1$ with the sampling rate of 1 sample per second over the range of 0 to $60.12 \mathrm{mmHg}$. Repeated measurements show close agreement which indicates a high level of repeatability of the sensor. This work shows that the proposed wireless LCP-based ICP sensor is able to operate in a pressure range of 0 to $50 \mathrm{mmHg}$ which is within the requirement for ICP monitoring for TBI patients.

\section{ACKNOWLEDGEMENTS}

This research has been funded by FY2016 Thesis Grant for Doctoral Degree Student under National Research Council of Thailand (NRCT) through Mahidol University. 


\section{REFERENCES}

[1] Kawoos U, McCarron RM, Auker CR, Chavko M., "Advances in intracranial pressure monitoring and its significance in managing traumatic brain injury," International Journal of Molecular Sciences; vol. 16(12), pp. 28979-97, 2015.

[2] Hyder AA, Wunderlich CA, Puvanachandra P, Gururaj G, Kobusingye OC. The impact of traumatic brain injuries: a global perspective. NeuroRehabilitation, vol. 22(5), pp. 341-53, 2007. [Online]. Available from: http://www.ncbi.nlm.nih.gov/pubmed/18162698

[3] Schwarzbold M, Diaz A, Martins ET, Rufino A, Amante LN, Thais ME, et al., "Psychiatric disorders and traumatic brain injury," Neuropsychiatric Disease and Treatment, vol. 4(4), pp. 797-816, 2008.

[4] Roytowski D, Figaji A., "Raised intracranial pressure: What it is and how to recognise it," Continuing Medical Education, vol. 31(11), pp. 390-5, 2013. [Online]. Available from: http://www.cmej.org.za/index.php/cmej/article/view/2909/3284.

[5] Harary M, Dolmans RGF, Gormley WB. Intracranial pressure monitoring-review and avenues for development. Sensors (Switzerland), vol. 18(2), pp. 3-7, 2018.

[6] Zhong J, Dujovny M, Park HK, Perez E, Perlin AR, Diaz FG. Advances in ICP monitoring techniques. Neurological Research, vol. 25(4), pp. 339-50, 2003. [Online]. Available from: http://www.tandfonline.com/doi/full/10.1179/016164103101201661

[7] Khan M, Shallwani H, Khan M, Shamim M., "Noninvasive monitoring intracranial pressure - A review of available modalities," Surgical Neurology International, vol. 8(1), p. 51, 2017. [Online]. Available from: http://surgicalneurologyint.com/surgicalint-articles/noninvasive-monitoring-intracranial-pressurea-review-of-available-modalities/

[8] Bashirullah R., "Wireless implants," IEEE Microwave Magazine, vol. 11(7 SUPPL.), pp. 14-23, 2010.

[9] Yu L, Kim BJ, Meng E., "Chronically implanted pressure sensors: Challenges and state of the field," Sensors (Switzerland), vol. 14(11), pp. 20620-44, 2014.

[10] Hodgins D, Bertsch A, Post N, Frischholz M, Volckaerts B, Spensley J, et al., "Healthy aims: Developing new medical implants and diagnostic equipment," IEEE Pervasive Computing, vol. 7(1), pp. 14-20, 2008.

[11] Joung YH., "Development of implantable medical devices: From an engineering perspective," International Neurourology Journal, vol. 17(3), pp. 98-106, 2013.

[12] Mapato M, Klysuban P, Kulworawanichpong T, Chomnawang N., "Metal-embedded SU-8 slab techniques for low-resistance micromachined inductors," International Journal of Electrical and Computer Engineering, vol. 8(6), pp. 4772-80, 2018.

[13] Khan MWA, Björninen T, Sydänheimo L, Ukkonen L., "Remotely Powered Piezoresistive Pressure Sensor: Toward Wireless Monitoring of Intracranial Pressure," IEEE Microwave and Wireless Components Letters, vol. 26(7), pp. 549-51, 2016.

[14] Khan MWA, Sydänheimo L, Ukkonen L, Björninen T., "Inductively Powered Pressure Sensing System Integrating a Far-Field Data Transmitter for Monitoring of Intracranial Pressure," IEEE Sensors Journal, vol. 17(7), pp. 2191-7, 2017.

[15] Behfar MH, Björninen T, Moradi E, Sydänheimo L, Ukkonen L. Biotelemetric Wireless Intracranial Pressure Monitoring: An In Vitro Study. International Journal of Antennas and Propagation, vol. 1-10, 2015.

[16] Wang F, Zhang X, Shokoueinejad M, Iskandar BJ, Medow JE, Webster JG., "A Novel Intracranial Pressure Readout Circuit for Passive Wireless LC Sensor," IEEE Transactions on Biomedical Circuits and Systems, vol. 11(5), pp. 1123-32, 2017.

[17] Cabello-ruiz R, Tecpoyotl-torres M, Jácome AT, Vera-dimas G, Koshevaya S, Vargas-chable P., "Displacement mechanical amplifiers designed on poly-silicon," International Journal of Electrical and Computer Engineering (IJECE), vol. 9(2), pp. 894-901, 2019.

[18] Meng X, Browne KD, Huang SM, Mietus C, Cullen DK, Tofighi MR, et al., "Dynamic evaluation of a digital wireless intracranial pressure sensor for the assessment of traumatic brain injury in a swine model," IEEE Transactions on Microwave Theory and Techniques, vol. 61(1), pp. 316-25, 2013.

[19] Jiang H, Guo Y, Wu Z, Zhang C, Jia W, Wang Z., "Implantable Wireless Intracranial Pressure Monitoring Based on Air Pressure Sensing," IEEE Transactions on Biomedical Circuits and Systems, vol. 12(5), pp. 1076-87, 2018.

[20] Sattayasoonthorn P, Suthakorn J, Chamnanvej S., "On the feasibility of a liquid crystal polymer pressure sensor for intracranial pressure measurement," Biomedical Engineering/Biomedizinische Technik, Mar 15. 2019, [Online]. Available from: http://www.degruyter.com/view/j/bmte.ahead-ofprint/bmt-2018-0029/bmt-2018-0029.xml 
[21] Sattayasoonthorn P, Suthakorn J, Chamnanvej S, Miao J, Kottapalli AGP., "LCP MEMS implantable pressure sensor for Intracranial Pressure measurement LCP MEMS implantable pressure sensor for Intracranial Pressure measurement," In: The 7th IEEE International Conference on Nano/Molecular Medicine and Engineering IEEE, p. 63-7, 2013. [Online]. Available from: http://ieeexplore.ieee.org/document/6766317/

[22] Wang X, Engel J, Liu C., "Liquid crystal polymer for MEMS: Processes and applications. Journal of Micromechanics and Microengineering; vol. 13, pp. 628-633, 2003. [Online]. Available from: http://www.iop.org/EJ/abstract/0960-1317/13/5/314.

[23] Bouneb I, Kerrour F., "Nanometric modelization of gas structure, multidimensional using comsol software," International Journal of Electrical and Computer Engineering, vol. 8(4), pp. 2014-20, 2018.

[24] Vogt C, Reber J, Waltisberg D, Buthe L, Marjanovic J, Munzenrieder N, et al. "A wearable bluetooth le sensor for patient monitoring during MRI scans," Proceedings of the Annual International Conference of the IEEE Engineering in Medicine and Biology Society, EMBS. 2016 pp. 4975-8, Oct 2016.

[25] Stephen NG., "On energy harvesting from ambient vibration," Journal of Sound and Vibration, vol. 293(1-2), pp. 409-25, 2006.

[26] Nanda A, Karami MA. "Energy harvesting from arterial blood pressure for powering embedded micro sensors in human brain," Journal of Applied Physics, vol. 121(12), 2017. 